\title{
Article
}

\section{Prone Position in Mechanically Ventilated COVID-19 Patients: A Multicenter Study}

\author{
Richard Vollenberg ${ }^{1, *(\mathbb{D}}$, Philipp Matern ${ }^{1}$, Tobias Nowacki ${ }^{1,2}$, Valentin Fuhrmann ${ }^{1}$, Jan-Sören Padberg ${ }^{3}$, \\ Kevin Ochs ${ }^{1}{ }^{1}$, Katharina Schütte-Nütgen ${ }^{4}$, Markus Strauß ${ }^{3,5}$, Hartmut Schmidt ${ }^{1}$ and Phil-Robin Tepasse ${ }^{1, *}$ \\ 1 Department of Medicine B, Gastroenterology, Hepatology, Endocrinology, Clinical Infectiology, \\ University Hospital Muenster, 48149 Muenster, Germany; p.matern@outlook.de (P.M.); \\ Tobias.Nowacki@ukmuenster.de (T.N.); Valentin.Fuhrmann@ukmuenster.de (V.F.); \\ Kevin.Ochs@ukmuenster.de (K.O.); Hartmut.Schmidt@ukmuenster.de (H.S.) \\ 2 Department of Medicine, Gastroenterology, Marienhospital Steinfurt, 48565 Steinfurt, Germany \\ 3 Department of Cardiology I-Coronary and Peripheral Vascular Disease, Heart Failure, \\ University Hospital Muenster, 48149 Muenster, Germany; jan-soeren.padberg@ukmuenster.de (J.-S.P.); \\ Markus.Strauss@ukmuenster.de (M.S.) \\ 4 Department of Medicine D, Nephrology, University Hospital Muenster, 48149 Muenster, Germany; \\ Katharina.Schuette-Nuetgen@ukmuenster.de \\ 5 Department of Cardiology, Sector Preventive Medicine, Health Promotion, Faculty of Health, \\ School of Medicine, University Witten/Herdecke, 58095 Hagen, Germany \\ * Correspondence: richardvollenberg@outlook.de or Richard.vollenberg@ukmuenster.de (R.V.); \\ Phil-Robin.Tepasse@ukmuenster.de (P.-R.T.)
}

\section{check for} updates

Citation: Vollenberg, R.; Matern, P.; Nowacki, T.; Fuhrmann, V.; Padberg, J.-S.; Ochs, K.; Schütte-Nütgen, K.; Strauß, M.; Schmidt, H.; Tepasse, P.-R. Prone Position in Mechanically Ventilated COVID-19 Patients: A Multicenter Study. J. Clin. Med. 2021, 10, 1046. https://doi.org/10.3390/ jcm10051046

Academic Editors: Francesco Pugliese and Francesco Alessandri

Received: 26 January 2021

Accepted: 24 February 2021

Published: 3 March 2021

Publisher's Note: MDPI stays neutral with regard to jurisdictional claims in published maps and institutional affiliations.

Copyright: (c) 2021 by the authors. Licensee MDPI, Basel, Switzerland. This article is an open access article distributed under the terms and conditions of the Creative Commons Attribution (CC BY) license (https:// creativecommons.org/licenses/by/ $4.0 /)$.
Abstract: Background: The prone position (PP) is increasingly used in mechanically ventilated coronavirus disease 2019 (COVID-19) acute respiratory distress syndrome (ARDS) patients. However, studies investigating the influence of the PP are currently lacking in these patients. This is the first study to investigate the influence of the PP on the oxygenation and decarboxylation in COVID-19 patients. Methods: A prospective bicentric study design was used, and in mechanically ventilated COVID-19 patients, $\mathrm{PP}$ was indicated from a partial pressure of oxygen in arterial blood $\left(\mathrm{P}_{\mathrm{aO}}\right) /$ fraction of inspired oxygen $\left(\mathrm{F}_{\mathrm{IO} 2}\right)$ ratio of $<200$. Patients were left prone for $16 \mathrm{~h}$ each. Pressure levels, $\mathrm{F}_{\mathrm{IO} 2}$, were adjusted to ensure a $\mathrm{P}_{\mathrm{aO} 2}$ greater than $60 \mathrm{mmHg}$. Blood gas analyses were performed before (baseline $0.5 \mathrm{~h})$, during $(1 / 2 / 5.5 / 9.5 / 13 \mathrm{~h})$, and after being in the PP $(1 \mathrm{~h})$, the circulatory/ventilation parameters were continuously monitored, and lung compliance (LC) was roughly calculated. Responders were defined compared to the baseline value $\left(\mathrm{P}_{\mathrm{aO} 2} / \mathrm{F}_{\mathrm{IO} 2}\right.$ ratio increase of $\geq 15 \%$; partial pressure of carbon dioxide $\left(\mathrm{P}_{\mathrm{aCO}}\right)$ decrease of $\left.\geq 2 \%\right)$. Results: 13 patients were included and $36 \mathrm{PP}$ sessions were conducted. Overall, $\mathrm{P}_{\mathrm{aO} 2} / \mathrm{F}_{\mathrm{IO} 2}$ increased significantly in the PP $(p<0.001)$. Most $\mathrm{P}_{\mathrm{aO} 2} / \mathrm{F}_{\mathrm{IO} 2}$ responders $(29 / 36 \mathrm{PP}$ sessions, $77 \%)$ were identified $9.5 \mathrm{~h}$ after turning prone (14\% slow responders), while most $\mathrm{P}_{\mathrm{aCO} 2}$ responders (15/36 PP sessions, $42 \%$ ) were identified $13 \mathrm{~h}$ after turning prone. A subgroup of patients (interval intubation to $\mathrm{PP} \geq 3$ days) showed less $\mathrm{P}_{\mathrm{aO} 2} / \mathrm{F}_{\mathrm{IO} 2}$ responders ( $16 \%$ vs. $77 \%$ ). An increase in $\mathrm{P}_{\mathrm{aCO} 2}$ and minute ventilation in the PP showed a significant negative correlation $\left(p<0.001\right.$ ). $\mathrm{LC}$ (median before the $\mathrm{PP}=38 \mathrm{~mL} / \mathrm{cm} \mathrm{H}_{2} \mathrm{O}$; two patients with $\mathrm{LC}>80 \mathrm{~mL} / \mathrm{cm} \mathrm{H}_{2} \mathrm{O}$ ) showed a significant positive correlation with the 28 day survival of patients $(p=0.01)$. Conclusion: The PP significantly improves oxygenation in COVID-19 ARDS patients. The data suggest that they also benefit most from an early PP. A decrease in minute ventilation may result in fewer $\mathrm{P}_{\mathrm{aCO} 2}$ responders. LC may be a predictive outcome parameter in COVID-19 patients. Trial registration: Retrospectively registered.

Keywords: prone position; COVID-19 ARDS; lung compliance

\section{Background}

Severe acute respiratory syndrome coronavirus 2 (SARS-CoV-2) is a new virus first identified in December 2019 in Wuhan, China. It can lead to severe pneumonia and 
respiratory failure (i.e., coronavirus disease 2019 (COVID-19)). Since its identification, the virus has been spreading rapidly across the world. In March 2020, the WHO declared COVID-19 as a pandemic. In February 2021, over 110 million confirmed cases and over 2.4 million confirmed deaths across 215 countries were counted. Mild courses of the disease were observed in $81 \%$ of patients, while severe disease courses were found in $14 \%$, and $5 \%$ of patients became critically ill and were admitted to an intensive care unit (ICU) [1]. The reasons for admission to an ICU are usually dyspnoea, tachypnea, and hypoxemia, with radiological evidence of pulmonary infiltrates [2,3]. SARS-CoV-2 patients can develop acute respiratory distress syndrome (ARDS). ARDS is a type of respiratory failure characterized by poor oxygenation and pulmonary infiltrates [4]. Currently there is no evidence proven in disease-specific medical treatment available. Current intensive care treatment for COVID19 ARDS is mostly in line with ARDS recommendations. In patients with early disease, therapy with remdesivir can be evaluated $[5,6]$. Corticosteroids in critically ill patients can reduce mortality [7]. In patients with severe ARDS and no response to conservative ARDS therapy, VV-ECMO therapy can be evaluated (e.g., $\mathrm{P}_{\mathrm{aO} 2} / \mathrm{FIO} 2<70 \mathrm{mmHg}$ ). For this purpose, ECMO therapy should be evaluated early in the course of disease in patients with severe respiratory failure [8]. Since the 1970s, the prone position (PP) has been used in ARDS patients to treat severe hypoxemia $[9,10]$. PP means repositioning the patient $180^{\circ}$ from the supine position. The PP changes the respiratory mechanics with a reduction in the pleural pressure gradient, a reduction in tidal hyperinflation, ventilation-associated lung damage, and mobilization of secretions [11]. The PP is increasingly used in COVID19 patients with mild-to-severe ARDS. However, studies on the influence of the PP in SARS-CoV-2 patients are currently lacking. This study investigated the influence of the PP on oxygenation, decarboxylation, and the further course of the disease in COVID-19 ARDS patients.

\section{Methods}

After approval by the institutional human ethics committee (2020-245-f-S; 04/2020), adult patients with a positive polymerase chain reaction (PCR) SARS-CoV-2 nasopharyngeal swab were identified, as previously described [9]. Most patients had a viral load quantification (Ct value at diagnosis, $<30$ ), and some patients had only a positive PCR result. In this bicentric, retrospective study, COVID-19 patients admitted to Münster University Hospital $(n=38)$ and to Hospital Steinfurt $(n=34)$ were screened from February to May 2020. ARDS was defined according to the WHO/Berlin definition [12]. Included patients showed bilateral SARS-CoV-2 typical infiltrates on chest X-ray or computed tomography, patients with respiratory failure due to heart failure or volume overload were excluded, and moderate-to-severe oxygenation impairment had to be present. ARDS severity was defined by the ratio of arterial oxygen tension to fraction of inspired oxygen $\left(\mathrm{P}_{\mathrm{aO} 2} / \mathrm{F}_{\mathrm{IO} 2}\right)$. Mild ARDS was defined as $200 \mathrm{mmHg}<\mathrm{P}_{\mathrm{aO} 2} / \mathrm{F}_{\mathrm{IO} 2} \leq 300 \mathrm{mmHg}$ (positive end-expiratory pressure (PEEP) or continuous positive airway pressure (CPAP) $\geq 5 \mathrm{cmH}_{2} \mathrm{O}$ ), moderate ARDS as $100 \mathrm{mmHg}<\mathrm{P}_{\mathrm{aO} 2} / \mathrm{F}_{\mathrm{IO} 2} \leq 200 \mathrm{mmHg}\left(\mathrm{PEEP} \geq 5 \mathrm{cmH}_{2} \mathrm{O}\right)$, and severe ARDS as $\mathrm{P}_{\mathrm{aO} 2} / \mathrm{F}_{\mathrm{IO} 2} \leq 100 \mathrm{mmHg}\left(\mathrm{PEEP} \geq 5 \mathrm{cmH}_{2} \mathrm{O}\right.$ ) [11]. All included patients met the WHO severe acute respiratory infection (SARI) definition (respiratory infection with a history of fever, cough, onset within the past 10 days, and required hospitalization) [13]. In 13 patients with moderate or severe ARDS, the PP was performed due to severe hypoxemia (Figure S1). In the following, PP sessions were evaluated independently of the patients $(n=36$ PP sessions). The PP was performed in patients with a partial pressure of oxygen in arterial blood $\left(\mathrm{P}_{\mathrm{aO} 2}\right)$ / fraction of inspired oxygen $\left(\mathrm{F}_{\mathrm{IO} 2}\right)$ ratio of $\leq 200$ in both hospitals. The abdominal positions were performed in both study centers with respect to the same protocol.

Patients were sedated and mechanically ventilated under pressure control (respirators: Evita 4, Dräger, Lübeck, Germany; C3, Hamilton Medical, Bonaduz, Switzerland). No PP was performed in ARDS patients with a contraindication (abdomen apertum, spinal instability, increased intra-abdominal pressure, threatening cardiac arrhythmias, or overt shock). Patients with veno-arterial/veno-veno extracorporeal membrane oxygenation 
(VA-/VV-ECMO) therapy were excluded from the study for the duration of the ECMO therapy ( $n=3$ patients, $6 \mathrm{PP}$ sessions). Pressure levels and positive end-expiratory pressure (PEEP) were adjusted to individual pressure volume relationships and the fraction of inspired oxygen $\left(\mathrm{F}_{\mathrm{IO} 2}\right)$ to ensure a $\mathrm{P}_{\mathrm{aO} 2}$ greater than $60 \mathrm{mmHg}$. PEEP was set according to the ARDS network $\mathrm{F}_{\mathrm{IO} 2} / \mathrm{PEEP}$ table (higher PEEP/lower $\mathrm{F}_{\mathrm{IO} 2}$ ) [9]. A target tidal volume of $6 \mathrm{~mL} / \mathrm{kg}$ ideal body weight was used in both study centers. The patients were left in the $\mathrm{PP}$ for $16 \mathrm{~h}$ each. The decision to repeat the PP was made individually for each patient depending on the response to the therapy and the overall clinical condition. Patients received continuous sedation and analgetic therapy. The ventilator settings were not changed $30 \mathrm{~min}$ before the PP. Arterial blood gas analyses (ABL800 FLEX, Radiometer, Krefeld, Germany) were performed before (baseline, $0.5 \mathrm{~h}$ ), during $(1,2,5.5,9.5$, and $13 \mathrm{~h})$, and after $(1 \mathrm{~h})$ the PP. The circulatory and ventilator settings were continuously monitored (PEEP, peak pressure $\left(\mathrm{P}_{\mathrm{Max}}\right)$, tidal volume, and lung compliance). The ventilation parameters were left unchanged in the first hour of the PP, except for $\mathrm{F}_{\mathrm{IO} 2}, \mathrm{PEEP}$, and $\mathrm{P}_{\mathrm{Max}}$. The $\mathrm{P}_{\mathrm{aO} 2} / \mathrm{F}_{\mathrm{IO} 2}$ ratio (mmHg) during the PP sessions $(1,2,5.5,9.5$, and $13 \mathrm{~h}$ ) were used to assess oxygenation for the study period.

Patients treated with the PP were classified as either $\mathrm{P}_{\mathrm{aO} 2}$ or partial pressure of carbon dioxide $\left(\mathrm{P}_{\mathrm{aCO}}\right)$ responders. The $\mathrm{P}_{\mathrm{aO} 2} / \mathrm{F}_{\mathrm{IO} 2}$ responders were defined as having $\mathrm{a} \geq 15 \%$ increase in the $\mathrm{P}_{\mathrm{aO} 2} / \mathrm{F}_{\mathrm{IO} 2}$ ratio $(\mathrm{mmHg})$ [14], while the $\mathrm{P}_{\mathrm{aCO}}$ responders were defined as having a decrease of $\geq 2 \% \mathrm{PaCO}_{\mathrm{aCO}}$ compared to the baseline value ( $0.5 \mathrm{~h}$ before the PP). $\mathrm{P}_{\mathrm{aO} 2} / \mathrm{F}_{\mathrm{IO} 2}$ and $\mathrm{P}_{\mathrm{aCO} 2}$ responders were determined at $1,2,5.5,9.5$, and $13 \mathrm{~h}$ in the PP. The identified $\mathrm{P}_{\mathrm{aO} 2} / \mathrm{F}_{\mathrm{IO} 2}$ responders were divided into fast responders $\left(\mathrm{P}_{\mathrm{aO} 2} / \mathrm{F}_{\mathrm{IO} 2}>15 \%\right.$ in the first $2 \mathrm{~h}$ during the $\mathrm{PP})$ and slow responders $\left(\mathrm{P}_{\mathrm{aO} 2} / \mathrm{F}_{\mathrm{IO} 2}>15 \%\right.$ after $5.5 / 9.5 \mathrm{~h}$ in the PP) [15-17].

The lung compliance (LC) was calculated using the following formula: $\mathrm{LC}=$ tidal volume $\left(\mathrm{V}_{\mathrm{T}}\right) /$ pressure difference $(\Delta \mathrm{p})=\mathrm{V}_{\mathrm{T}} /\left(\mathrm{P}_{\mathrm{Max}}-\mathrm{PEEP}\right)$ [18]. A normal LC is approximately $200 \mathrm{~mL} / \mathrm{cm} \mathrm{H}_{2} \mathrm{O}$.

In this study, data are given as the median and the range. To compare changes in the supine/prone position, a non-parametric one-way ANOVA for repeated measures (KruskalWallis test) was used. Dunn's multiple comparison post hoc test was used to compare pairs of time points. Correlations of at least ordinally scaled parameters were examined using Spearman's rank correlation, while correlations of nominally/metrically scaled parameters were performed using the eta correlation coefficient. Calculations were performed using statistical software (SPSS, IBM SPSS Statistics, New York, USA). Differences with a $p$-level less than 0.05 were considered statistically significant.

\section{Results}

The study included 13 COVID-19 patients with ARDS from Münster University Hospital and Marienhospital Steinfurt. Characterization of the patient population is shown in Table 1. All patients had suspected or confirmed bacterial superinfection, and all patients received antibiotic therapy. The median time period from intubation to PP was 0 days, interquartile range (IQR) 0; 2 (Table 2). The number of PP sessions per patient ranged from 1 to 6 , and 7 of the 13 patients died within 28 days after the last PP ( 28 day mortality, equal ICU mortality and equal hospital mortality). The last nasopharyngeal swab before the first pp is shown (Table 2). There was a significant correlation between viral load in deep nasopharyngeal swabs and 28 day mortality (eta coefficient, $r=0.950 ; p=0.01 ; n=7$ ). 
Table 1. Characterization of the severe acute respiratory syndrome coronavirus 2 (SARS-CoV-2) acute respiratory distress syndrome (ARDS) patient population, including cardiovascular risk factors and other pre-existing conditions, pre-medication, and co-infections.

\begin{tabular}{|c|c|c|c|c|}
\hline \multirow[t]{2}{*}{$n=13$} & \multicolumn{4}{|c|}{ Characterization of the Patient Population } \\
\hline & Min. & Max. & Median (Q1; Q3) & Avg. $( \pm$ SD) \\
\hline Age (years) & 45 & 78 & $63(51 ; 69)$ & $61( \pm 10.2)$ \\
\hline Body weight (kg) & 72 & 140 & $80(76 ; 98)$ & $88( \pm 19.8)$ \\
\hline BMI $\left(\mathrm{kg} / \mathrm{m}^{2}\right)$ & 22 & 36 & $25(25 ; 30)$ & $27( \pm 4.4)$ \\
\hline \multicolumn{2}{|c|}{ Cardiovascular risk factors } & \multicolumn{3}{|c|}{ Pre-medication } \\
\hline & $n(\%)$ & & & $n(\%)$ \\
\hline Arterial hypertension & $6(46 \%)$ & Immuno & ppressive therapy & $0(0)$ \\
\hline Diabetes mellitus & $0(0 \%)$ & Antihy & rtensive therapy & $6(46 \%)$ \\
\hline Nicotine abuse & $4(30.6 \%)$ & & E-inhibitor & $1(7.7 \%)$ \\
\hline \multirow[t]{2}{*}{ Male gender } & $12(92.3 \%)$ & AT & -antagonists & $3(23.1 \%)$ \\
\hline & & & lin therapy & $0(0 \%)$ \\
\hline \multicolumn{2}{|c|}{ Other pre-existing conditions } & \multirow{2}{*}{\multicolumn{2}{|c|}{$\begin{array}{l}\text { Oral diabetes therapy } \\
\text { Systemic steroids therapy }\end{array}$}} & $0(0 \%)$ \\
\hline & $n(\%)$ & & & $0(0)$ \\
\hline Cardiovascular diseases & $5(38.5 \%)$ & \multicolumn{2}{|c|}{ Local steroids therapy } & $0(0)$ \\
\hline Cerebrovascular diseases & $1(7.7 \%)$ & \multirow{2}{*}{\multicolumn{2}{|c|}{ Antiviral medication }} & $2(15.4 \%)$ \\
\hline COPD & $0(0 \%)$ & & & \\
\hline Bronchial asthma & $0(0 \%)$ & \multicolumn{3}{|c|}{ Co-infections } \\
\hline Other pulmonary diseases & $0(0 \%)$ & & & $n(\%)$ \\
\hline Immunosuppressive disease & $2(15.4 \%)$ & \multicolumn{2}{|c|}{ Bacterial superinfection } & $13(100 \%)$ \\
\hline Comorbidity & $12(92.3 \%)$ & \multicolumn{2}{|c|}{ Mycotic superinfection } & $3(23.1 \%)$ \\
\hline Medication before illness & $12(92.3 \%)$ & \multicolumn{2}{|c|}{ Antibiotic therapy (ICU) } & $13(100 \%)$ \\
\hline
\end{tabular}

Q1, quartile 1; Q3, quartile 3; Avg., average; SD, standard deviation; BMI, body mass index, COPD, chronic obstructive pulmonary disease, ACE-inhibitor, angiotensin-converting enzyme inhibitor; AT1R-antagonists, angiotensin II receptor type 1 antagonists; ICU, intensive care unit.

\subsection{Prone Position Responder: Oxygenation and Decarboxylation}

Considering the $\mathrm{P}_{\mathrm{aO} 2} / \mathrm{F}_{\mathrm{IO} 2}$ ratio after $1 \mathrm{~h}$ in the $\mathrm{PP}$, responders were identified in 24/36 (67\%) PP sessions (cutoff value: $>15 \%$ increase in $\mathrm{P}_{\mathrm{aO} 2} / \mathrm{F}_{\mathrm{IO} 2}$ ) (Tables 2 and 3 and Figure 1). The median improvement of oxygenation $\left(\mathrm{P}_{\mathrm{aO} 2} / \mathrm{F}_{\mathrm{IO} 2}\right)$ was $38.4 \%[26 \% ; 95 \%]$. The number of $\mathrm{PP}$ sessions that had an improvement in oxygenation $\left(\mathrm{P}_{\mathrm{aO} 2} / \mathrm{F}_{\mathrm{IO} 2}>15 \%\right)$ continued to increase up until $9.5 \mathrm{~h}$ in the PP. Considering the entire period in the PP, $29 / 36$ (81\%) responders were identified: $24 / 36$ fast responders, $5 / 36$ slow responders, and $7 / 36$ nonresponders were identified. After turning prone, a significant median improvement (responders and nonresponders) in $\mathrm{P}_{\mathrm{aO} 2} / \mathrm{F}_{\mathrm{IO} 2}$ was achieved at the $1,2,5.5,9.5$, and $13 \mathrm{~h}$ time points $(p<0.001$; Figure 2$)$. The maximum increase in the $\mathrm{P}_{\mathrm{aO} 2} / \mathrm{F}_{\mathrm{IO} 2}$ ratio of the responders was observed after $5.5 \mathrm{~h}$ in the PP (median 58.3\% [31\%; 95\%]). In $3 / 13$ patients, the interval from intubation to the first PP was three days or more $(3,9$, and 16 days). Considering this subgroup, oxygenation improved significantly (cutoff: $\geq 15 \%$ ) only in $1 / 6$ PP sessions ( $16 \%$ vs. $77 \%)$. 
Table 2. Demographic and clinical characteristics of the patients.

\begin{tabular}{|c|c|c|c|c|c|c|c|c|c|c|c|c|c|}
\hline $\begin{array}{l}\text { Patient } \\
\text { Number }\end{array}$ & Age & Gender & $\begin{array}{l}\text { Charlson } \\
\text { Comorbidity } \\
\text { Index (CCI) }\end{array}$ & $\begin{array}{c}\text { Severity of } \\
\text { ARDS: } \\
\mathrm{P}_{\mathrm{aO} 2} / \mathrm{F}_{\mathrm{IO} 2} \\
\text { Ratio on The } \\
\text { Day of Prone }\end{array}$ & $\begin{array}{l}\text { Intubation } \\
\text { to Prone } \\
\text { Ventilation } \\
\text { in Days }\end{array}$ & $\begin{array}{l}\text { Number of } \\
\text { Prone } \\
\text { Ventilation } \\
\text { Sessions }\end{array}$ & $\begin{array}{c}\mathrm{P}_{\mathrm{aO} 2} / \mathrm{F}_{\mathrm{IO} 2} \\
\text { Responder } \\
\text { (1 h PP) }\end{array}$ & $\begin{array}{c}\mathrm{P}_{\mathrm{aCO} 2} \\
\text { Responder } \\
\text { (1 h PP) }\end{array}$ & $\begin{array}{c}\text { Viral } \\
\text { Load: Ct } \\
\text { Value } \\
\text { (RT-PCR) }\end{array}$ & $\begin{array}{c}\text { PEEP } \\
\text { Reduction } \\
(1 \mathrm{~h} \mathrm{PP})\end{array}$ & $\begin{array}{c}P_{\text {Max }} \\
\text { Reduction } \\
(1 \mathrm{~h} \mathrm{PP})\end{array}$ & $\begin{array}{c}\text { Minute } \\
\text { Ventilation } \\
\text { Reduction } \\
\text { (1 h PP) }\end{array}$ & $\begin{array}{c}28 \text { Day } \\
\text { Mortality } \\
\text { after Last } \\
\text { PP }\end{array}$ \\
\hline 1 & 65 & Male & 4 & 102 & 0 & 4 & $4 / 4$ & $0 / 4$ & 27.4 & $1 / 4$ & $2 / 4$ & $3 / 4$ & Died \\
\hline 2 & 68 & Male & 3 & 128 & 9 & 3 & $1 / 3$ & $1 / 3$ & 35.6 & $1 / 3$ & $0 / 3$ & $2 / 3$ & Survived \\
\hline 3 & 50 & Male & 1 & 145 & 0 & 2 & $2 / 2$ & $0 / 2$ & 22.7 & $0 / 2$ & $1 / 2$ & $1 / 2$ & Died \\
\hline 4 & 60 & Male & 3 & 97 & 0 & 2 & $2 / 2$ & $0 / 2$ & 0 & $0 / 2$ & $1 / 2$ & $2 / 2$ & Survived \\
\hline 6 & 63 & Male & 3 & 61 & 0 & 1 & $1 / 1$ & $1 / 1$ & - & $1 / 1$ & $0 / 1$ & $1 / 1$ & Died \\
\hline 7 & 63 & Male & 3 & 99 & 0 & 2 & $2 / 2$ & $1 / 2$ & - & $2 / 2$ & $0 / 2$ & $2 / 2$ & Survived \\
\hline 8 & 70 & Male & 4 & 200 & 3 & 2 & $0 / 2$ & $1 / 2$ & 35.1 & $0 / 2$ & $2 / 2$ & $1 / 2$ & Survived \\
\hline 9 & 45 & Male & 0 & 88 & 0 & 3 & $0 / 3$ & $1 / 3$ & 0 & $1 / 3$ & $1 / 3$ & $2 / 3$ & Survived \\
\hline 10 & 77 & Male & 4 & 124 & 1 & 5 & $4 / 5$ & $3 / 5$ & - & $1 / 5$ & $3 / 5$ & $4 / 5$ & Died \\
\hline 11 & 58 & Male & 2 & 127 & 16 & 1 & $0 / 1$ & $0 / 1$ & - & $0 / 1$ & $1 / 1$ & $0 / 1$ & Survived \\
\hline 12 & 78 & Male & 4 & 115 & 1 & 2 & $1 / 2$ & $0 / 2$ & - & $0 / 2$ & $0 / 2$ & $2 / 2$ & Died \\
\hline 13 & 48 & Female & 1 & 58 & 1 & 6 & $4 / 6$ & $1 / 6$ & - & $0 / 6$ & $1 / 6$ & $4 / 6$ & Died \\
\hline
\end{tabular}

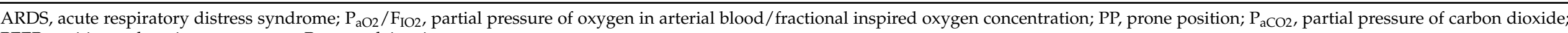
PEEP, positive end-expiratory pressure; $P_{\max }$, peak inspiratory pressure. 


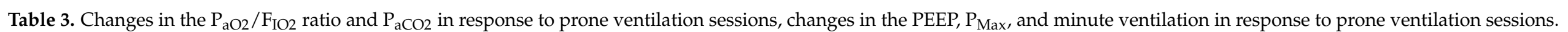

\begin{tabular}{|c|c|c|c|c|c|c|}
\hline \multicolumn{7}{|c|}{$\mathrm{P}_{\mathrm{aO} 2} / \mathrm{F}_{\mathrm{IO} 2}$} \\
\hline & $1 \mathrm{~h} \mathrm{PP}$ & 2 h PP & $5.5 \mathrm{~h} \mathrm{PP}$ & $9.5 \mathrm{~h} \mathrm{PP}$ & $13 \mathrm{~h}$ PP & $1 \mathrm{~h} \mathrm{SP}$ \\
\hline Responder $(\geq 15 \%)$ & $24 / 36(67 \%)$ & $24 / 36(67 \%)$ & $26 / 36(72 \%)$ & $29 / 36(81 \%)$ & $28 / 36(78 \%)$ & $20 / 36(56 \%)$ \\
\hline M [Q1; Q3] increase (responder) & $38.4 \%[26 \% ; 95 \%]$ & $42.6 \%[23 \% ; 84 \%]$ & $58.3 \%[31 \% ; 95 \%]$ & $40.9 \%[28 \% ; 67 \%]$ & $48 \%[36 \% ; 81 \%]$ & $46.6 \%[30 \% ; 62 \%]$ \\
\hline Avg. ( \pm SD) increase (responder) & $69.7 \%( \pm 67 \%)$ & $62.8 \%( \pm 58 \%)$ & $68.7 \%( \pm 52 \%)$ & $55.8 \%( \pm 49 \%)$ & $65.7 \%( \pm 50 \%)$ & $52 \%( \pm 32 \%)$ \\
\hline PP1 & $9 / 13(69 \%)$ & $12 / 13(92 \%)$ & $11 / 13(85 \%)$ & $11 / 13(85 \%)$ & $12 / 13(92 \%)$ & $11 / 13(85 \%)$ \\
\hline PP2 & $8 / 11(73 \%)$ & $6 / 11(55 \%)$ & $8 / 11(73 \%)$ & $9 / 11(82 \%)$ & $8 / 11(73 \%)$ & $4 / 11(36 \%)$ \\
\hline PP3 & $4 / 6(67 \%)$ & $3 / 6(50 \%)$ & $3 / 6(50 \%)$ & $5 / 6(83 \%)$ & $5 / 6(83 \%)$ & $5 / 6(83 \%)$ \\
\hline PP4 & $3 / 3(100 \%)$ & $1 / 3(33 \%)$ & $3 / 3(100 \%)$ & $3 / 3(100 \%)$ & $2 / 3(66 \%)$ & $0 / 3(0 \%)$ \\
\hline PP6 & $0 / 1(0 \%)$ & $0 / 1(0 \%)$ & $0 / 1(0 \%)$ & $0 / 1(0 \%)$ & $0 / 1(0 \%)$ & $0 / 1(0 \%)$ \\
\hline \multicolumn{7}{|c|}{$\mathbf{P}_{\mathrm{aCO} 2}$} \\
\hline & $1 \mathrm{~h} \mathrm{PP}$ & 2 h PP & 5.5 h PP & $9.5 \mathrm{~h} \mathrm{PP}$ & $13 \mathrm{~h}$ PP & $1 \mathrm{~h} \mathrm{SP}$ \\
\hline Responder $(\geq 2 \%)$ & $12 / 36(33 \%)$ & $14 / 36(39 \%)$ & $12 / 36(33 \%)$ & $12 / 36(33 \%)$ & $15 / 36(42 \%)$ & $15 / 36(42 \%)$ \\
\hline M [Q1; Q3] increase (responder) & $\begin{array}{c}-4.5 \% \\
{[-27 \% ;-3 \%]}\end{array}$ & $\begin{array}{c}-9 \% \\
{[-21 \% ;-5 \%]}\end{array}$ & $\begin{array}{c}-12 \% \\
{[-26 \% ;-9 \%]}\end{array}$ & $\begin{array}{c}-9 \% \\
{[-21 \% ;-4 \%]}\end{array}$ & $\begin{array}{c}-11 \% \\
{[-21 \% ;-7 \%]}\end{array}$ & $\begin{array}{c}-11 \% \\
{[-25 \% ;-9 \%]}\end{array}$ \\
\hline Avg. ( \pm SD) increase (responder) & $-11.8 \%( \pm 13.1 \%)$ & $-13.3 \%( \pm 11.8 \%)$ & $-16.5 \%( \pm 12.6 \%)$ & $-15.5 \%( \pm 17 \%)$ & $-16.3 \%( \pm 14.6 \%)$ & $-18.9 \%( \pm 16 \%)$ \\
\hline PP1 & $6 / 13(46 \%)$ & $7 / 13(54 \%)$ & $7 / 13(54 \%)$ & $7 / 13(54 \%)$ & $6 / 13(46 \%)$ & $7 / 13(54 \%)$ \\
\hline PP4 & $1 / 3(33 \%)$ & $1 / 3(33 \%)$ & $0 / 3(0 \%)$ & $0 / 3(0 \%)$ & $1 / 3(33 \%)$ & $0 / 3(0 \%)$ \\
\hline PP5 & $1 / 2(50 \%)$ & $1 / 2(50 \%)$ & $2 / 2(100 \%)$ & $2 / 2(100 \%)$ & $2 / 2(100 \%)$ & $1 / 2(50 \%)$ \\
\hline PP6 & $0 / 1(0 \%)$ & $0 / 1(0 \%)$ & $0 / 1(0 \%)$ & $0 / 1(0 \%)$ & $1 / 0(100 \%)$ & $0 / 1(0 \%)$ \\
\hline \multicolumn{7}{|c|}{ PEEP } \\
\hline & $1 \mathrm{~h} \mathrm{PP}$ & & $5.5 \mathrm{~h} \mathrm{PP}$ & & & $1 \mathrm{~h} \mathrm{SP}$ \\
\hline Reduction & $8 / 36(22 \%)$ & & $8 / 36(22 \%)$ & & & $15 / 36(42 \%)$ \\
\hline $\mathrm{M}[\mathrm{Q} 1 ; \mathrm{Q} 2]$ decrease & $-14.2 \%[-22 \% ;-8 \%]$ & & $-13.8 \%[-21 \% ;-8 \%]$ & & & $-19.9 \%[-30 \% ;-12 \%]$ \\
\hline Avg. $( \pm S D)$ & $-16.2 \%( \pm 9 \%)$ & & $-15.1 \%( \pm 7.8 \%)$ & & & $-21.4 \%( \pm 12.2 \%)$ \\
\hline PP1 & $2 / 13(15 \%)$ & & $2 / 13(15 \%)$ & & & $4 / 13(31 \%)$ \\
\hline PP2 & $1 / 11(9 \%)$ & & $3 / 11(27 \%)$ & & & $4 / 11(36 \%)$ \\
\hline PP3 & $3 / 6(50 \%)$ & & $1 / 6(17 \%)$ & & & $3 / 6(50 \%)$ \\
\hline PP4 & $2 / 3(67 \%)$ & & $2 / 3(67 \%)$ & & & $2 / 3(67 \%)$ \\
\hline PP5 & $0 / 2(0 \%)$ & & $0 / 2(0 \%)$ & & & $2 / 2(100 \%)$ \\
\hline PP6 & $0 / 0(0 \%)$ & & $0 / 2(0 \%)$ & & & $0 / 1(0 \%)$ \\
\hline
\end{tabular}


Table 3. Cont.

\begin{tabular}{|c|c|c|c|}
\hline \multicolumn{4}{|c|}{$P_{\text {Max }}$} \\
\hline & $1 \mathrm{~h} \mathrm{PP}$ & $5.5 \mathrm{~h}$ PP & $1 \mathrm{~h} \mathrm{SP}$ \\
\hline Reduction & $15 / 36(42 \%)$ & $14 / 36(39 \%)$ & $12 / 36(33 \%)$ \\
\hline M [Q1; Q2] decrease & $-3.9 \%[-14 \% ;-3 \%]$ & $-7.4 \%[-12 \% ;-6 \%]$ & $-22.6 \%[-27 \% ;-10 \%]$ \\
\hline Avg. $( \pm$ SD) & $-7.7 \%( \pm 6.6 \%)$ & $-9.3( \pm 5.9 \%)$ & $-18.8 \%( \pm 9.5 \%)$ \\
\hline PP1 & $4 / 13(31 \%)$ & $5 / 13(38 \%)$ & $4 / 13(31 \%)$ \\
\hline PP2 & $6 / 11(55 \%)$ & $4 / 11(36 \%)$ & $2 / 11(18 \%)$ \\
\hline PP3 & $2 / 6(33 \%)$ & $3 / 6(50 \%)$ & $4 / 6(67 \%)$ \\
\hline PP4 & $2 / 3(67 \%)$ & $1 / 3(33 \%)$ & $1 / 3(33 \%)$ \\
\hline PP6 & $0 / 1(0 \%)$ & $0 / 1(0 \%)$ & $0 / 1(0 \%)$ \\
\hline \multicolumn{4}{|c|}{ Minute ventilation } \\
\hline & $1 \mathrm{~h} \mathrm{PP}$ & $5.5 \mathrm{~h}$ PP & $1 \mathrm{~h} \mathrm{SP}$ \\
\hline Reduction & $25 / 36(69 \%)$ & $27 / 36(75 \%)$ & $21 / 36(58 \%)$ \\
\hline M [Q1; Q2] decrease & $-15.8 \%[-23 \% ;-6 \%]$ & $-19 \%[-32 \% ;-8 \%]$ & $-18.5 \%[-35 \% ;-6 \%]$ \\
\hline Avg. $( \pm \mathrm{SD})$ & $-18.7 \%( \pm 17.3 \%)$ & $-22.4 \%( \pm 17.5 \%)$ & $-21.5 \%( \pm 10.6 \%)$ \\
\hline PP1 & $7 / 13(54 \%)$ & $8 / 13(62 \%)$ & $9 / 13(69 \%)$ \\
\hline PP2 & $10 / 11(91 \%)$ & $9 / 11(82 \%)$ & $5 / 11(45 \%)$ \\
\hline PP4 & $2 / 3(67 \%)$ & $3 / 3(100 \%)$ & $3 / 3(100 \%)$ \\
\hline PP5 & $1 / 2(50 \%)$ & $0 / 2(0 \%)$ & $1 / 2(50 \%)$ \\
\hline PP6 & $1 / 1(100 \%)$ & $1 / 1(100 \%)$ & $1 / 1(100 \%)$ \\
\hline
\end{tabular}

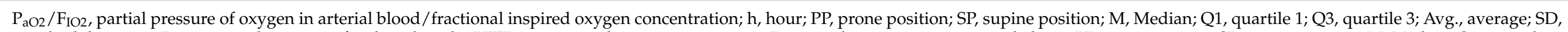

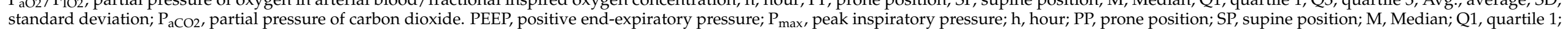

Q3, quartile 3; Avg., average; SD, standard deviation. 


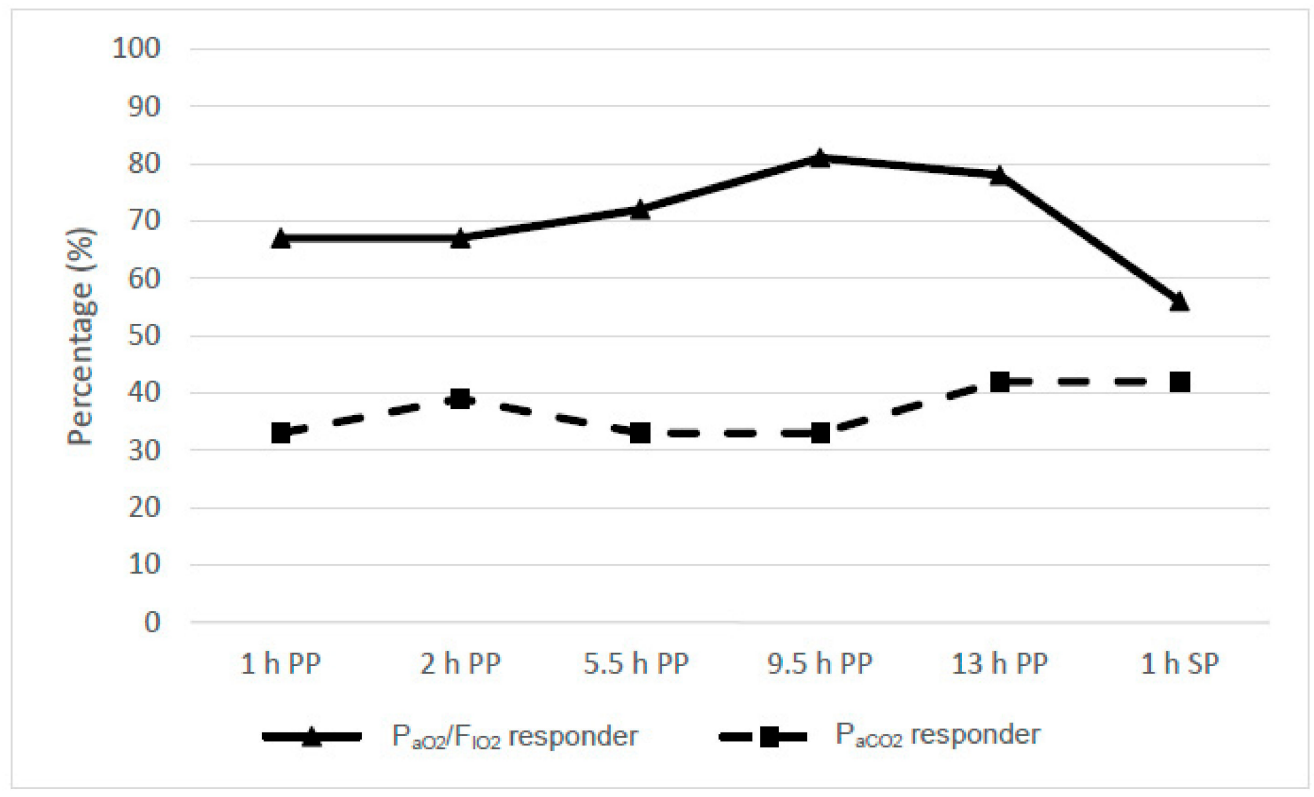

Figure 1. Partial pressure of oxygen in arterial blood/fractional inspired oxygen concentration $\left(\mathrm{P}_{\mathrm{aO} 2} / \mathrm{F}_{\mathrm{IO} 2}\right)$ responder $\left(\geq 15 \%\right.$ improvement in $\left.\mathrm{P}_{\mathrm{aO} 2} / \mathrm{F}_{\mathrm{IO} 2}\right)$ and $\mathrm{P}_{\mathrm{aCO} 2}$ responder $(\geq 2 \%$ improvement in $\mathrm{P}_{\mathrm{aCO} 2}$ ) during the prone (PP) and supine (SP) positions. $h$, hour.

Subjects treated with PP sessions were classified as either $\mathrm{P}_{\mathrm{aO} 2}$ or $\mathrm{P}_{\mathrm{aCO} 2}$ responders (Tables 2 and $3 \mathrm{~A}$ and Figures 1 and 2). A significant decrease in $\mathrm{P}_{\mathrm{aCO} 2}$ was observed in $12 / 36(33 \%)$ PP sessions ( $1 \mathrm{~h})$. The median decarboxylation of the responders improved by $4.5 \%[-27 \% ;-3 \%]$. Most $\mathrm{P}_{\mathrm{aCO} 2}$ responders were identified after $13 \mathrm{~h}$ in the PP $(42 \%)$. This corresponds to three PP sessions with a slow improvement in decarboxylation. The maximum improvement in decarboxylation was seen in the PP after $5.5 \mathrm{~h}$ (median $-4.5 \%$ [-26\%; $-9 \%]$ ) (Figure 1). One hour after turning in the supine position, a persistent increase in $\mathrm{P}_{\mathrm{aCO} 2}$ was observed in $15 / 36(42 \%)$ sessions. At this time point, the responders showed a median decrease of $11 \%[-25 \% ;-9 \%]$. Overall, the $\mathrm{P}_{\mathrm{aCO} 2}$ in patients showed no significant change ( $p=0.809$; Figure 2$)$.

\subsection{Invasive Ventilation of COVID-19 Patients in the Prone Position}

In $8 / 36$ (22\%) PP sessions, a PEEP reduction was possible ( 1 and $5.5 \mathrm{~h}$ in the PP) (Table 3 and Figure 2). After $1 \mathrm{~h}$ in the PP, the median PEEP reduction was $14.2 \%[-22 \%$; $-8 \%]$; after $5.5 \mathrm{~h}$, the median reduction was $13.8 \%[-21 \% ;-8 \%]$. A reduction in the peak pressure $\left(\mathrm{P}_{\max }\right)$ was possible after $1 \mathrm{~h}$ in the $\mathrm{PP}$ in $15 / 36$ cases $(42 \%$; median $-3.9 \%[-14 \% ;-3 \%])$ and after $5.5 \mathrm{~h}$ in $14 / 36$ cases $(39 \%$; median $-7.4 \%$ [ $-12 \%$; $-6 \%]$ ). After supine rotation, a persistent reduction in the peak pressure was observed in $12 / 36$ cases (33\%; median $-22.6 \%$ [ $-27 \%$; $-10 \%]$ ). The maximum of the median PEEP and peak reduction could be reached $1 \mathrm{~h}$ after turning to the supine position. Moreover, 25/36 (69\%) after $1 \mathrm{~h}$ versus $27 / 36(75 \%)$ after $5.5 \mathrm{~h}$ showed a reduction in minute ventilation (median $-15.86[-23 \% ;-6 \%]$ versus median -19 $[-32 \% ;-8 \%]$ ). In the supine position (after $1 \mathrm{~h}$ ), there was a persistent reduction in minute ventilation in $21 / 36$ cases (58\%). The maximum reduction of minute ventilation was observed at $5.5 \mathrm{~h}$ in the PP.

This study also showed a significant negative correlation between the percentage increase in $\mathrm{PaCO}_{\mathrm{aC}}$ and the percentage change in minute ventilation in the PP (Spearman's rank correlation: $r=-0.616, p \leq 0.001, n=36$ ). There was no significant correlation between the minute ventilation and the PEEP (Spearman's rank correlation: $r=-0.09, p=0.601$, $n=36$ ) or the $\mathrm{P}_{\mathrm{Max}}$ (Spearman's rank correlation: $r=-0.188, p=0.271, n=36$ ) in the PP sessions. 

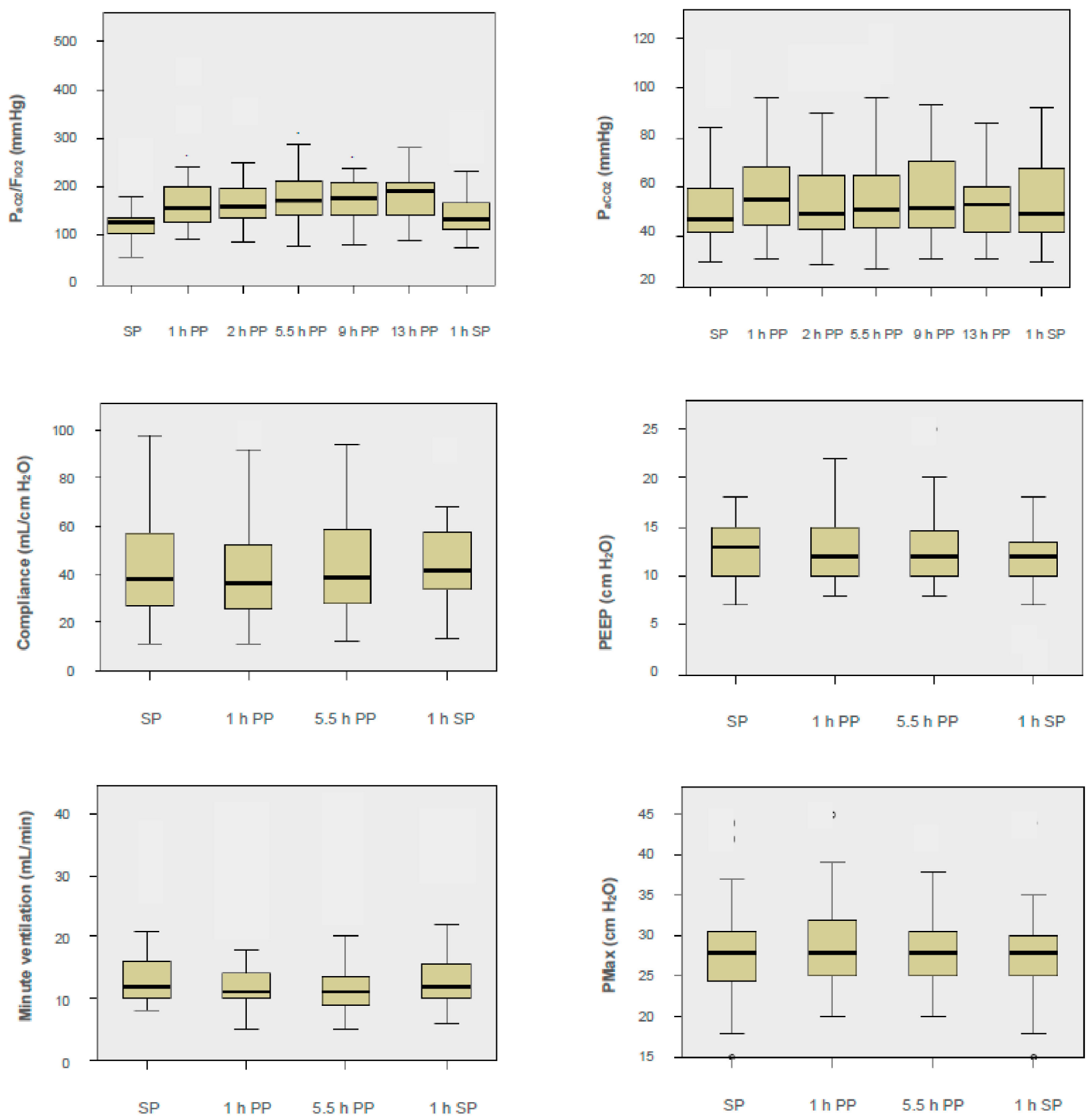

Figure 2. $\mathrm{P}_{\mathrm{aO} 2} / \mathrm{F}_{\mathrm{IO} 2}, \mathrm{P}_{\mathrm{aCO} 2}$, lung compliance, minute ventilation, peak inspiratory pressure $\left(\mathrm{P}_{\mathrm{Max}}\right)$, and positive endexpiratory pressure (PEEP) before and during the prone and supine positions.

\subsection{Lung Compliance in COVID-19 Patients Correlates with 28 Day Survival}

The LC $\left(\Delta \mathrm{p}=\mathrm{V}_{\mathrm{T}} /\left(\mathrm{P}_{\mathrm{Max}}-\mathrm{PEEP}\right)\right)$ was calculated $0.5 \mathrm{~h}$ before the PP, 1 and $5.5 \mathrm{~h}$ during the $\mathrm{PP}$, and $1 \mathrm{~h}$ after rotation in the supine position again (Figure 2). In terms of median, an LC of $38 \mathrm{~mL} / \mathrm{cm} \mathrm{H}_{2} \mathrm{O}\left[26 \mathrm{~mL} / \mathrm{cm} \mathrm{H}_{2} \mathrm{O} ; 58 \mathrm{~mL} / \mathrm{cm} \mathrm{H}_{2} \mathrm{O}\right.$ ] was found before the patient was turned prone (Figure 2). One hour after rotation, the median $\mathrm{LC}$ was $36 \mathrm{~mL} / \mathrm{cm}$ $\mathrm{H}_{2} \mathrm{O}\left[25 \mathrm{~mL} / \mathrm{cm} \mathrm{H}_{2} \mathrm{O} ; 53 \mathrm{~mL} / \mathrm{cm} \mathrm{H}_{2} \mathrm{O}\right]$, which was $39 \mathrm{~mL} / \mathrm{cm} \mathrm{H}_{2} \mathrm{O}\left[27 \mathrm{~mL} / \mathrm{cm} \mathrm{H}_{2} \mathrm{O}\right.$; $59 \mathrm{~mL} / \mathrm{cm} \mathrm{H}_{2} \mathrm{O}$ ] after $5.5 \mathrm{~h}$. After a further turn to the supine position $(1 \mathrm{~h})$, the median LC of $42 \mathrm{~mL} / \mathrm{cm} \mathrm{H}_{2} \mathrm{O}\left[33 \mathrm{~mL} / \mathrm{cm} \mathrm{H}_{2} \mathrm{O} ; 58 \mathrm{~mL} / \mathrm{cm} \mathrm{H}_{2} \mathrm{O}\right.$ ] was unchanged. Therefore, it can be concluded that in terms of the median, there was a reduced LC in the COVID-19 patients. LC did not change significantly in patients during proning $(p>0.05)$. Two patients had an $\mathrm{LC}$ of $>80 \mathrm{~mL} / \mathrm{cm} \mathrm{H}_{2} \mathrm{O}\left(84 \mathrm{~mL} / \mathrm{cm} \mathrm{H}_{2} \mathrm{O}, 98 \mathrm{~mL} / \mathrm{cm} \mathrm{H}_{2} \mathrm{O}\right)$. The LC ( $1 \mathrm{~h}$ before the PP) 
showed a significant correlation with patient survival (28 day mortality: Eta coefficient, $r=0.418 ; p=0.011)$.

\section{Discussion}

Since 1974, the PP has been the subject of numerous studies in ARDS patients. Most patients placed in the PP exhibit mild-to-dramatic improvements in oxygenation, regardless of lung injury categorization. There are multiple factors that may influence oxygenation in the PP by reducing ventilation and perfusion mismatches, the intrapulmonary shunt, and ventilator-induced lung injury (VILI) [14]. These changes are due to a reduction in dependent atelectasis and pleural pressure gradient [19], to alveolar recruitment [20], to homogeneous ventilation [21], and to a reduction in lung compression by the heart and abdomen [22-24].

The PP in the treatment of therapy-resistant oxygenation disorders in ARDS patients may be most effective when initiated early during the exudative phase. At this point, congestive and compressive atelectasis develop, whereas in the intermediate phase of ARDS (more than one week), fibrosis and type II cell hyperplasia are more prevalent [25-27]. In this study, the interval from intubation to the first PP was three days or more $(3,9$, and 16 days) in $3 / 13$ patients. Considering this subgroup, oxygenation improved (cutoff: $\geq 15 \%$ ) only in $1 / 6$ (16\%, $1 \mathrm{~h}$ PP) PP sessions versus 23/30 (77\%) when the interval from intubation to PP was less than three days. These results suggest that ARDS patients with COVID-19 also benefit most from an early PP (less than three days from intubation to prone ventilation). The 28 day mortality after the last PP session was 54\% (7/13 patients died). In studies since 2010, the overall rates of mortality in ARDS patients in an ICU were shown to be $38 \%$ and the 28 day mortality to be $30 \%$ [28]. In a subgroup of ARDS with H1N1 viral infection, a mortality of 36-38\% has been reported [29]. In comparison to H1N1, as well as a meta-analysis of ARDS patients, there was a significantly increased mortality rate in our SARS-CoV-2 patient cohort.

\subsection{Prevalence of Positive Oxygenation Response in COVID-19 Patients}

In this bicentric, retrospective observational study, 29/36 PP sessions (81\%) showed significant improvement in the $\mathrm{P}_{\mathrm{aO} 2} / \mathrm{F}_{\mathrm{IO} 2}$ ratio $(\geq 15 \%, 9.5 \mathrm{~h} \mathrm{PP})$. The proportion of responders to prone positioning reported in different studies varies depending on the selected cutoff values for oxygenation improvement, the selected time points (comparison supine position with $\mathrm{PP}$ ), and the number of subjects sampled. In most studies, cutoff values for an increase in $\mathrm{P}_{\mathrm{aO} 2}$ or $\mathrm{P}_{\mathrm{aO} 2} / \mathrm{F}_{\mathrm{IO} 2}$ of $10-20 \mathrm{mmHg}$ or an increase of $10-20 \%$ have been used to define responders to prone positioning [14]. In approximately $20 \%$ of studies, an improvement in oxygenation was found in less than $70 \%$ of patients. In the majority of previous studies (approximately 50\%), an improvement of 70-85\% was achieved in patients. Moreover, $30 \%$ of the studies found a significant improvement in oxygenation in $\geq 90 \%$ of ARDS patients in the PP [14]. The time required to improve oxygenation in the PP differs in ARDS patients. In spontaneously breathing patients with COVID-19 with severe hypoxemic respiratory failure, the PP when awake is associated with improved oxygenation [30-34]. In this study, compared to the baseline, positive responders were identified after $1 \mathrm{~h}$ in 24/36 (67\%) PP sessions. This is a low rate of responders compared to previous ARDS studies ( $80 \%$ of the studies had $>70 \%$ responders). When considering all control points, most responders were identified $9.5 \mathrm{~h}$ after turning prone $(29 / 36 ; 78 \%)$. This is the average number of responders compared to other studies. In 5/36 PP sessions, there was a slow increase in oxygenation (17\% slow responders). The majority of patients experienced a rapid improvement in oxygenation in $1 \mathrm{~h}$. These observations are consistent with previous ARDS data [35,36]. On average, $\mathrm{P}_{\mathrm{aO} 2}$ increases of $23-78 \mathrm{mmHg}$ and improvements in oxygenation of $34-62 \%$ are seen in responders to the PP [14]. In this study, $\mathrm{P}_{\mathrm{aO} 2} / \mathrm{F}_{\mathrm{IO} 2}$ responders showed a maximum increase $5.5 \mathrm{~h}$ after rotation to the PP. The median oxygenation improved by $58.3 \%$ [31\%; $95 \%$ ]. In comparison to previous studies, there was a good-to-above average improvement in oxygenation in $\mathrm{P}_{\mathrm{aO} 2} / \mathrm{F}_{\mathrm{IO} 2}$ responders. 


\subsection{Alveolar Ventilation in COVID-19 Patients: Prevalence of Positive Decarboxylation Response}

The effect of the $\mathrm{PP}$ on $\mathrm{PaCO}_{\mathrm{aC}}$ in ARDS patients has been described inconsistently in clinical studies. Various factors have been identified to have an influence on the elimination of $\mathrm{CO}_{2}$ in the PP. Patients in the PP can experience both increased and reduced elimination of $\mathrm{CO}_{2}$. Most observational studies have shown no improvement in $\mathrm{P}_{\mathrm{aCO} 2}$ clearance in patients in the PP. These results are surprising, because in the PP, an improvement in the ventilation/perfusion ratio of the lungs $\left(\mathrm{V}_{\mathrm{A}} / \mathrm{Q}\right)$ and a reduced intrapulmonary shunt from recruitment leads to improved physiological dead-space fraction [37]. An increase in $\mathrm{PaCO}_{\mathrm{aCO}}$ in the PP may depend on a decreased alveolar ventilation and a relative change in lung perfusion [15]. A reduced elasticity of the thorax in the PP with a consecutively reduced breathing volume and minute ventilation can lead to an increase in $\mathrm{P}_{\mathrm{aCO}}$ in pressure control ventilation [4]. An increase in $\mathrm{CO}_{2}$ elimination appears to be associated with an increase in the net recruitment of lung sections [38]. An increased dead-space fraction is a feature of the early phase of ARDS. It has been shown that an increased dead-space fraction is associated with an increased risk of death [39]. A decrease in $\mathrm{PaCO}_{\mathrm{aC}}$ in the $\mathrm{PP}$ is predictive of improved outcome in ARDS [15,40].

Several investigators have classified patients treated with the $\mathrm{PP}$ in $\mathrm{P}_{\mathrm{aCO} 2}$ responders and nonresponders. Cutoff values for a $\mathrm{P}_{\mathrm{aCO} 2}$ decrease differ from 1 to $2 \mathrm{mmHg}$ [15-17]. One hour after turning in the $\mathrm{PP}$, in $12 / 36(33 \%) \mathrm{PP}$ sessions, a significant $\mathrm{P}_{\mathrm{aCO} 2}$ response $\left(\mathrm{P}_{\mathrm{aCO}}\right.$ decrease of $\geq 2 \%$ ) was detected. Most of the values above the cutoff were found in the PP after $13 \mathrm{~h}(15 / 36,42 \%)$. Compared to previous ARDS studies (53-54\% responders), fewer responders were identified in SARS-CoV-2 patients $[12,13]$. After $1 \mathrm{~h}$ in the PP, responders had a median $\mathrm{P}_{\mathrm{aCO} 2}$ reduction of $4.5 \%$ [-27\%; $\left.-3 \%\right]$. The maximum $\mathrm{P}_{\mathrm{aCO} 2}$ reduction in $\mathrm{P}_{\mathrm{aCO}}$ responders was observed after $5.5 \mathrm{~h}$ in the PP (median $-12 \%[-26 \%$; $-9 \%])$. These findings are in agreement with previous ARDS data [15].

Regarding PEEP, $\mathrm{P}_{\mathrm{Max}}$, and minute ventilation, no significant differences could be found in the PP. There was a significant negative correlation between the percentage increase in $\mathrm{P}_{\mathrm{aCO}}$ and the percentage change in minute ventilation in the $\mathrm{PP}(p<0.001)$. A reduction in minute ventilation can result in an increase in $\mathrm{P}_{\mathrm{aCO} 2}$ in the PP in ARDS patients [4]. There was no significant correlation between minute ventilation and PEEP $(p=0.601)$ or $\mathrm{P}_{\mathrm{Max}}(p=0.271)$ in the PP. The results of this study suggest that a decrease in minute ventilation in ARDS patients with COVID-19 leads to fewer $\mathrm{P}_{\mathrm{aCO}}$ responders.

\subsection{Significant Correlation between Lung Compliance and Survival in COVID-19 Patients}

$\mathrm{LC}$ is the change in lung volume for a given pressure. Before turning to the PP, the patients showed a significantly reduced LC (median $38 \mathrm{~mL} / \mathrm{cm} \mathrm{H}_{2} \mathrm{O}\left[26 \mathrm{~mL} / \mathrm{cm} \mathrm{H}_{2} \mathrm{O}\right.$; $\left.\left.58 \mathrm{~mL} / \mathrm{cm} \mathrm{H}_{2} \mathrm{O}\right]\right)$. During the PP, there was no significant change in LC $(p=0.804)$. The compliance of the respiratory system was similar to other cohorts of COVID-19 patients and to ARDS not related to COVID-19 [18,41,42]. LC was $>80 \mathrm{~mL} / \mathrm{cm} \mathrm{H}_{2} \mathrm{O}$ in two patients, confirming the thesis that there are some COVID-19 patients with severe ARDS and relatively high LC [43]. In our study, there was a significant positive correlation between LC and 28 day survival. To the best of our knowledge, this is the first study to show a significant correlation between LC and survival in ventilated COVID-19 patients. These results should be further examined and confirmed in subsequent, larger studies.

\section{Conclusions}

COVID-19 patients showed significant improvement in the $\mathrm{P}_{\mathrm{aO} 2} / \mathrm{F}_{\mathrm{IO} 2}$ ratio in PP sessions (17\% slow responders). Our data suggest that they benefit most from an early PP. Compared to previous ARDS studies, fewer $\mathrm{P}_{\mathrm{aCO}}$ responders $(42 \%)$ were identified in SARS-CoV-2 patients. $\mathrm{P}_{\mathrm{aCO} 2}$ increase and minute ventilation in the PP showed a significant negative correlation $(p<0.001)$. The results suggest that a decrease in minute ventilation in COVID-19 patients may result in fewer $\mathrm{P}_{\mathrm{aCO} 2}$ responders. The viral load and the 28 day mortality correlated significantly $(p=0.01)$. Patients showed a reduction in the median LC, and interestingly, there were some patients with relatively high LC, confirming that there 
are some COVID-19 patients with severe ARDS and relatively high LC. LC and 28 day survival showed a significant positive correlation $(p=0.01)$, indicating that LC may be a predictive parameter for disease course and outcome in COVID-19 patients. These results should be further examined and confirmed in subsequent, larger studies.

Supplementary Materials: The following are available online at https:/ / www.mdpi.com/2077-0 383/10/5/1046/s1, Figure S1: Study flowchart prone position (PP) in coronavirus disease 2019 (COVID-19) patients. ICU, intensive care unit; ARDS, acute respiratory distress syndrome.

Author Contributions: Conceptualization, R.V., P.-R.T., and H.S.; data curation, R.V. and P.M.; formal analysis, R.V. and P.-R.T.; investigation, R.V. and P.-R.T.; methodology, R.V., P.-R.T., and V.F.; supervision, H.S.; writing-original draft, R.V., P.-R.T., and V.F.; writing-review and editing, V.F., M.S., K.S.-N., T.N., J.-S.P., P.M., K.O., and H.S. All authors have read and agreed to the published version of the manuscript.

Funding: The authors received no funding for this work.

Institutional Review Board Statement: Approval was given by the institutional human ethics committee in Muenster (2020-245-f-S).

Informed Consent Statement: Not applicable.

Data Availability Statement: The datasets used and/or analyzed during the current study are available from the corresponding author on reasonable request.

Conflicts of Interest: The authors declare no conflict of interest.

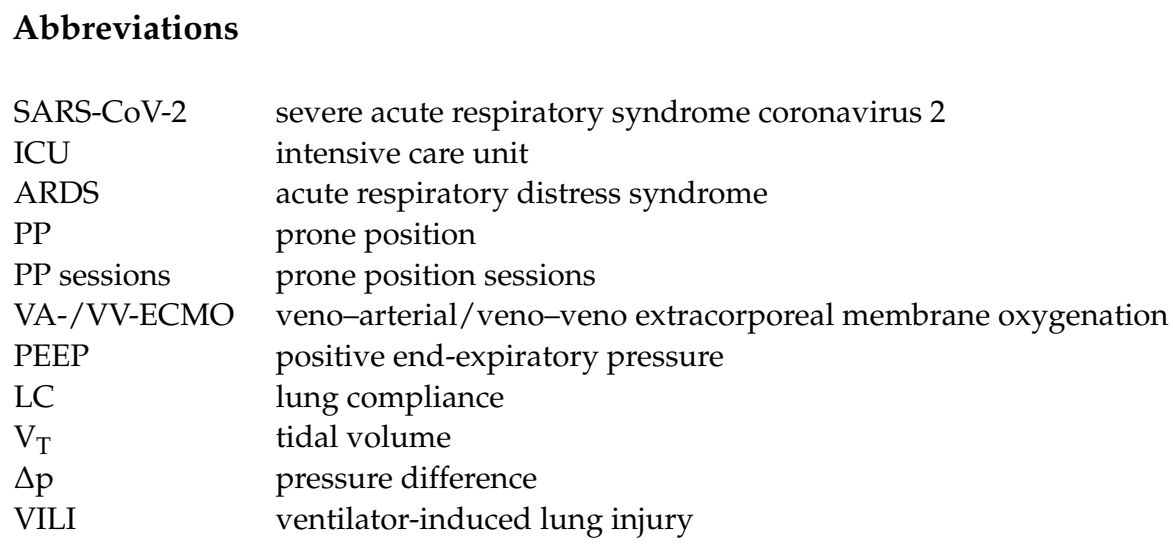

\section{References}

1. Wu, Z.; McGoogan, J.M. Characteristics of and Important Lessons from the Coronavirus Disease 2019 (COVID-19) Outbreak in China: Summary of a Report of 72,314 Cases from the Chinese Center for Disease Control and Prevention. JAMA 2020, 323, 1239-1242. [CrossRef] [PubMed]

2. Chung, M.; Bernheim, A.; Mei, X.; Zhang, N.; Huang, M.; Zeng, X.; Cui, J.; Xu, W.; Yang, Y.; Fayad, Z.A.; et al. CT Imaging Features of 2019 Novel Coronavirus (2019-nCoV). Radiology 2020, 295, 202-207. [CrossRef] [PubMed]

3. Kluge, S.; Janssens, U.; Welte, T.; Weber-Carstens, S.; Marx, G.; Karagiannidis, C. Empfehlungen zur inten-sivmedizinischen Therapie von Patienten mit COVID-19 (Recommendations for critically ill patients with COVID-19). Med. Klin. Intensivmed. Notf. 2020, 115, 175-177. [CrossRef]

4. Gattinoni, L.; Busana, M.; Giosa, L.; Macrì, M.M.; Quintel, M. Prone Positioning in Acute Respiratory Distress Syndrome. Semin. Respir. Crit. Care Med. 2019, 40, 094-100. [CrossRef] [PubMed]

5. Kluge, S.; Janssens, U.; Welte, T.; Weber-Carstens, S.; Schälte, G.; Spinner, C.D.; Malin, J.J.; Gastmeier, P.; Langer, F.; Wepler, M.; et al. S2k-Leitlinie-Empfehlungen zur stationären Therapie von Patienten mit COVID-19. Pneumology 2021, 75, 88-112. [CrossRef]

6. Horby, P.; Lim, W.S.; Emberson, J.R.; Mafham, M.; Bell, J.L.; Linsell, L.; Staplin, N.; Brightling, C.; Ustianowski, A.; Elmahi, E.; et al. Dexamethasone in Hospitalized Patients with Covid-19-Preliminary Report. N. Engl. J. Med. 2020. [CrossRef]

7. Beigel, J.H.; Tomashek, K.M.; Dodd, L.E.; Mehta, A.K.; Zingman, B.S.; Kalil, A.C.; Hohmann, E.; Chu, H.Y.; Luetkemeyer, A.; Kline, S.; et al. Remdesivir for the Treatment of Covid-19-Final Report. N. Engl. J. Med. 2020, 383, 1813-1826. [CrossRef] [PubMed]

8. MacLaren, G.; Combes, A.; Brodie, D. What's new in ECMO for COVID-19? Intensive Care Med. 2021, 47, 107-109. [CrossRef]

9. Piehl, M.A.; Brown, R.S. Use of extreme position changes in acute respiratory failure. Crit. Care Med. 1976, 4, 13-14. [CrossRef] 
10. Douglas, W.W.; Rehder, K.; Beynen, F.M.; Sessler, A.D.; Marsh, H.M. Improved oxygenation in patients with acute respiratory failure: The prone position. Am. Rev. Respir. Dis. 1977, 115, 559-566. [CrossRef] [PubMed]

11. Seeber, C. S3-Leitlinie Invasive Beatmung und Einsatz extrakorporaler Verfahren bei akuter respiratorischer Insuffizienz. 2017, 1295. Available online: https://www.awmf.org/uploads/tx_szleitlinien/001-021m_S3_Invasive_Beatmung_2017-12.pdf (accessed on 16 January 2021)

12. Force, A.D.T.; Ranieri, V.M.; Rubenfeld, G.D.; Thompson, B.T.; Ferguson, N.D.; Caldwell, E.; Fan, E.; Camporota, L.; Slutsky, A.S. Acute Respiratory Distress Syndrome. JAMA 2012, 307, 2526-2533. [CrossRef]

13. Bastos, L.S.; Niquini, R.P.; Lana, R.M.; Villela, D.A.M.; Cruz, O.G.; Coelho, F.C.; Codeço, C.T.; Gomes, M.F.C. COVID-19 e hospitalizações por SRAG no Brasil: Uma comparação até a $12^{a}$ semana epidemiológica de 2020. Cad. De Saúde Pública 2020, 36, e00070120. [CrossRef] [PubMed]

14. Kallet, R.H. A Comprehensive Review of Prone Position in ARDS. Respir. Care 2015, 60, 1660-1687. [CrossRef]

15. Gattinoni, L.; Vagginelli, F.; Carlesso, E.; Taccone, P.; Conte, V.; Chiumello, D.; Valenza, F.; Caironi, P.; Pesenti, A. Decrease in $\mathrm{P}_{\mathrm{aCO} 2}$ with prone position is predictive of improved outcome in acute respiratory distress syndrome*. Crit. Care Med. 2003, 31, 2727-2733. [CrossRef] [PubMed]

16. Lee, K.; Kim, M.-Y.; Yoo, J.-W.; Hong, S.-B.; Lim, C.-M.; Koh, Y. Clinical Meaning of Early Oxygenation Improvement in Severe Acute Respiratory Distress Syndrome under Prolonged Prone Positioning. Korean J. Intern. Med. 2010, 25, 58-65. [CrossRef]

17. Charron, C.; Repesse, X.; Bouferrache, K.; Bodson, L.; Castro, S.; Page, B.; Jardin, F.; Vieillard-Baron, A. PaCO2 and alveolar dead space are more relevant than $\mathrm{P}_{\mathrm{aO} 2} / \mathrm{F}_{\mathrm{iO} 2}$ ratio in monitoring the respiratory response to prone position in ARDS patients: A physiological study. Crit. Care 2011, 15, R175. [CrossRef] [PubMed]

18. Bos, L.D.J.; Paulus, F.; Vlaar, A.P.J.; Beenen, L.F.M.; Schultz, M.J. Subphenotyping Acute Respiratory Distress Syndrome in Patients with COVID-19: Consequences for Ventilator Management. Ann. Am. Thorac. Soc. 2020, 17, 1161-1163. [CrossRef] [PubMed]

19. Mutoh, T.; Guest, R.J.; Lamm, W.J.E.; Albert, R.K. Prone Position Alters the Effect of Volume Overload on Regional Pleural Pressures and Improves Hypoxemia in PigsIn Vivo. Am. Rev. Respir. Dis. 1992, 146, 300-306. [CrossRef]

20. Galiatsou, E.; Kostanti, E.; Svarna, E.; Kitsakos, A.; Koulouras, V.; Efremidis, S.C.; Nakos, G. Prone position augments recruitment and prevents alveolar overinflation in acute lung injury. Am. J. Respir. Crit. Care Med. 2006, 174, 187-197. [CrossRef]

21. Vieillard-Baron, A.; Rabiller, A.; Chergui, K.; Peyrouset, O.; Page, B.; Beauchet, A.; Jardin, F. Prone position improves mechanics and alveolar ventilation in acute respiratory distress syndrome. Intensiv. Care Med. 2004, 31, 220-226. [CrossRef] [PubMed]

22. Albert, R.K.; Hubmayr, R.D. The prone position eliminates compression of the lungs by the heart. Am. J. Respir. Crit. Care Med. 2000, 161, 1660-1665. [CrossRef]

23. Mure, M.; Glenny, R.W.; Domino, K.B.; Hlastala, M.P. Pulmonary gas exchange improves in the prone position with abdominal distension. Am. J. Respir. Crit. Care Med. 1998, 157, 1785-1790. [CrossRef] [PubMed]

24. Jardin, F. Role of the heart in the loss of aeration characterizing lower lobes in acute respiratory distress syndrome. Am. J. Respir. Crit. Care Med. 2001, 164, 171. [CrossRef]

25. Pelosi, P.; Brazzi, L.; Gattinoni, L. Prone position in acute respiratory distress syndrome. Eur. Respir. J. 2002, 20, 1017-1028. [CrossRef]

26. Blanch, L.; Mancebo, J.; Perez, M.; Martinez, M.; Mas, A.; Betbese, A.J.; Joseph, D.; Ballús, J.; Lucangelo, U.; Bak, E. Short-term effects of prone position in critically ill patients with acute respiratory distress syndrome. Intensiv. Care Med. 1997, 23, 1033-1039. [CrossRef]

27. Johannigman, J.A.; Davis, K.; Miller, S.L.; Campbell, R.S.; Luchette, F.A.; Frame, S.B.; Branson, R.D. Prone positioning for acute respiratory distress syndrome in the surgical intensive care unit: Who, when, and how long? Surgery 2000, 128, 708-716. [CrossRef]

28. Máca, J.; Jor, O.; Holub, M.; Sklienka, P.; Burša, F.; Burda, M.; Janout, V.; Ševčík, P. Past and present ARDS mortality rates: A systematic review. Respir. Care 2017, 62, 113-122. [CrossRef] [PubMed]

29. Sahoo, J.N.; Gurjar, M.; Mohanty, K.; Majhi, K.; Sradhanjali, G. Prone ventilation in H1N1 virus-associated severe acute respiratory distress syndrome: A case series. Int. J. Crit. Illn. Inj. Sci. 2019, 9, 182. [CrossRef]

30. Thompson, A.E.; Ranard, B.L.; Wei, Y.; Jelic, S. Prone positioning in awake, nonintubated patients with COVID-19 hypoxemic respiratory failure. JAMA Intern. Med. 2020, 17, e203030. [CrossRef]

31. Prasad, M.; Visrodia, K. Should I prone non-ventilated awake patients with COVID-19? Clevel. Clin. J. Med. 2020. [CrossRef]

32. Paul, V.; Patel, S.; Royse, M.; Odish, M.; Malhotra, A.; Koenig, S. Proning in non-intubated (pini) in times of COVID-19: Case series and a review. J. Intensiv. Care Med. 2020, 35, 818-824. [CrossRef]

33. Meng, L.; Qiu, H.; Wan, L.; Ai, Y.; Xue, Z.; Guo, Q.; Deshpande, R.; Zhang, L.; Meng, J.; Tong, C.; et al. Intubation and ventilation amid the COVID-19 outbreak. Anesthesiology 2020, 132, 1317-1332. [CrossRef] [PubMed]

34. Telias, I.; Katira, B.H.; Brochard, L. Is the prone position helpful during spontaneous breathing in patients with COVID-19? JAMA 2020, 323, 2265. [CrossRef] [PubMed]

35. Stocker, R.; Neff, T.; Stein, S.; Ecknauer, E.; Trentz, O.; Russi, E. Prone positioning and low-volume pressure-limited ventilation improve survival in patients with severe ARDS. Chest 1997, 111, 1008-1017. [CrossRef]

36. Papazian, L.; Paladini, M.-H.; Brégeon, F.; Huiart, L.; Thirion, X.; Saux, P.; Jammes, Y.; Auffray, J.-P. Is a short trial of prone positioning sufficient to predict the improvement in oxygenation in patients with acute respiratory distress syndrome? Intensiv. Care Med. 2001, 27, 1044-1049. [CrossRef] 
37. Kallet, R.H. Measuring dead-space in acute lung injury. Minerva Anestesiol. 2012, 78, 1297-1305. [PubMed]

38. Protti, A.; Chiumello, D.; Cressoni, M.; Carlesso, E.; Mietto, C.; Berto, V.; Lazzerini, M.; Quintel, M.; Gattinoni, L. Relationship between gas exchange response to prone position and lung recruitability during acute respiratory failure. Intensiv. Care Med. 2009, 35, 1011-1017. [CrossRef]

39. Nuckton, T.J.; Alonso, J.A.; Allet, R.I.H.K.; Daniel, B.M.; Pittet, J.-F.; Eisner, M.A.D.; Matthay, M.A. Pulmonary Dead-Space Fraction as a Risk Factor for Death in the Acute Respiratory Distress Syndrome. N. Engl. J. Med. 2002, 346, 1281-1286. [CrossRef]

40. Raurich, J.M.; Vilar, M.; Colomar, A.; Ibáñez, J.; Ayestarán, I.; Pérez-Bárcena, J.; Llompart-Pou, J.A. Prognostic value of the pulmonary dead-space fraction during the early and intermediate phases of acute respiratory distress syndrome. Respir. Care 2010, 55, 282-287. [PubMed]

41. Bellani, G.; Laffey, J.G.; Pham, T.; Fan, E.; Brochard, L.; Esteban, A.; Gattinoni, L.; Van Haren, F.; Larsson, A.; McAuley, D.F.; et al. Epidemiology, patterns of care, and mortality for patients with acute respiratory distress syndrome in intensive care units in 50 countries. JAMA 2016, 315, 788-800. [CrossRef]

42. Network, A.R.D.S.; Brower, R.G.; Matthay, M.A.; Morris, A.; Schoenfeld, D.; Thompson, B.T.; Wheeler, A. Ventilation with lower tidal volumes as compared with traditional tidal volumes for acute lung injury and the acute respiratory distress syndrome. N. Engl. J. Med. 2000, 342, 1301-1308. [CrossRef]

43. Li, X.; Ma, X. Acute respiratory failure in COVID-19: Is it typical ARDS? Crit. Care 2020, 24, 198. [CrossRef] [PubMed] 